\title{
Two Novel Iterative Joint Frequency-Offset and Channel Estimation Methods for OFDMA Uplink
}

\author{
Xiaoyu Fu, Hlaing Minn*, and C. D. Cantrell \\ Department of Electrical Engineering, The University of Texas at Dallas \\ e-mail: (\{xxf031000, hlaing.minn, cantrell $\} @$ utdallas.edu $)$
}

\begin{abstract}
We address joint estimation of frequency offsets and channel responses in OFDMA uplink. A cyclically equal-spaced, equal-energy interleaved pilot preamble is proposed by which two iterative estimators are developed. In the first estimator, we develop a modified SAGE (space alternating generalized expectation-maximization) method by incorporating multiuser interference cancellation in both time and frequency domains into the SAGE method. The proposed modified SAGE method yields a faster convergence rate, a better estimation performance, and a lower complexity than the existing conventional SAGE method from [10]. In the second method, we propose a very low complexity ad-hoc method to replace the high complexity frequency offset estimator of the first method. The proposed adhoc method is developed based on the time-domain characteristics of the pilot preamble and the frequency-domain power concentration property of OFDMA systems. It achieves almost the same estimation performance as the first proposed method but requires significantly lower complexity. Both proposed methods are not only better in estimation performance, convergence rate, and complexity, but also more robust to the number of active uplink users than the existing method while requiring only one OFDM training symbol and no restriction of the data subcarrier assignment scheme.
\end{abstract}

\section{INTRODUCTION}

Orthogonal Frequency Division Multiple Access (OFDMA) has been recently proposed or adopted in many industry standards (e.g., IEEE 802.16[1], 802.16e, 802.20, [2]). OFDMA has several advantages such as multi-user diversity, scalibity/adaptability in modulation/data rate/spectral occupancy, modularity, and low complexity equalization. But OFDMA uplink is associated with a more difficult task of synchronization and channel estimation due to the different time and frequency offsets of uplink users. Although timing offsets can be limited within the interference-free interval of the cyclic prefix by means of downlink synchronization or initial ranging process [3], (residual) frequency offsets result in inter-carrier interference (ICI), and could degrade the channel estimation and BER performance significantly [4]-[5]. Hence, reliable estimation of multiuser (residual) frequency offsets and channels is critical in realizing the OFDMA advantages.

Several methods have recently been proposed for the frequency offset estimation and/or channel estimation in OFDMA uplink [6]-[10]. However, they are associated with one or more of the following unfavorable requirements: very high complexity, several OFDM training symbols, specific subcarrier assignment scheme (CAS), ideal system situation, and a small number of SSs. In this paper, aiming at addressing the above drawbacks, we propose two iterative joint estimators utilizing a cyclically equal-spaced, equal-energy interleaved pilot preamble which is based on the best channel identification conditions [11]. In the first estimator, we develop a modified SAGE (MSAGE) method by incorporating multiuser interference cancellation in both time and frequency domains into the SAGE method. In the second method, we propose a very low complexity ad hoc method to replace the high complexity frequency offset estimator of the first method. The proposed ad hoc method is developed based on the timedomain characteristics of the pilot preamble and the frequencydomain power concentration property of OFDMA systems. It achieves almost the same estimation performance as the first proposed method but requires significantly lower complexity. Both proposed methods are not only better in estimation performance, convergence rate, and complexity, but also more robust to the number of active uplink users than the existing methods while requiring only one OFDM training symbol and no restriction of the data CAS.

Notations: The superscripts $T, *$ and $H$ represent the transpose, the conjugate and the Hermitian transpose, respectively. $\lfloor\cdot\rfloor$ is floor operation. $\operatorname{diag}\{\boldsymbol{x}\}$ is a diagonal matrix with the vector $\boldsymbol{x}$ in its main diagonal. $E[\cdot], \Re(\cdot)$, and $\|\cdot\|$ represent the expectation, the real part, and the Euclidean norm, respectively. $\boldsymbol{I}_{N}$ is the $N \times N$ identical matrix and $\mathbf{0}_{m}$ is the all-zero $m \times 1$ vector. $\bmod (i, K)$ denotes $i$ modulo $K$. $\mathfrak{B}_{\Phi_{k}}\{\boldsymbol{Z}\}$ represents the operation which returns a $B N_{p} \times N_{\text {col }}$ matrix by picking $B N_{p}$ rows from the $N \times N_{\text {col }}$ matrix $Z$ according to the indices of non-zero elements in the main diagonal of $\boldsymbol{\Phi}_{k}$.

\section{Signal Model and Preamble Design}

We consider uplink of an OFDMA system with $N$ subcarriers. Each uplink packet contains one OFDM training symbol (preamble) for synchronization and channel estimation followed by several data symbols. Each OFDM symbol has $N_{t}=N+N_{g}$ samples where $N$ is the length of useful part and $N_{g}$ is the length of cyclic prefix (CP) part. For data transmission, the $N$ sub-carriers are grouped into $Q$ subchannels. Each subchannel has $P$ subcarriers where $Q P \leq N$. We assume that there are $K$ SSs communicating with the BS simultaneously where $K \leq Q$. The $k$-th SS $(k \in\{1, \ldots, K\})$ has its own frequency offset $v_{k}$ (normalized by the subcarrier spacing), channel impulse response (CIR) $\overline{\boldsymbol{h}}_{k}$ and timing offset $d_{k}$. We 
assume that each SS has already performed at least integer frequency offset estimation and correction from the downlink. Hence, $\left\{v_{k}\right\}$ are modeled as independent uniformly distributed random variables with the range $[-0.5,0.5]$. The timing offset $d_{k} \in\left\{0, \ldots, d_{\max }-1\right\}$ is due to the timing estimation error in initial ranging process where $d_{\max }$ is the maximum possible timing estimation error determined by the initial ranging process [1]. $\overline{\boldsymbol{h}}_{k}=\left[\bar{h}_{k}(0), \bar{h}_{k}(1), \ldots, \bar{h}_{k}(L-1)\right]^{T}$ is the sample-spaced CIR vector where $L$, representing the maximum number of the sample-spaced channel taps, is the same for all SSs. The CIR vectors are assumed unchanged during a packet interval. To avoid inter-symbol interference (ISI), $N_{g}$ is chosen to be greater than or equal to $L^{\prime}=$ $L+d_{\max }$.

We use an interleaved CAS for the preamble where each SS is assigned a disjoint set of $N_{p}$ cyclically equal-spaced, equal-energy pilots. The pilot symbol on the $m$-th subcarrier of the $k$-th SS is given by

$X_{k}(m)= \begin{cases}a_{k} \mathcal{C}_{k}(i), & m=i D+\Delta_{k} ; \quad\left(i=0, \ldots, N_{p}-1\right) \\ 0, & \text { otherwise }\end{cases}$

where $a_{k}$ is the amplitude factor and $\Delta_{k}=\left\lfloor\frac{(k-1) D}{K}\right\rfloor$ is the subcarrier index shift for the $k$-th SS. Our pilot design sets the inter-user adjacent pilot tone spacings as large as possible. For simplicity, $\left\{a_{k}\right\}_{k=1}^{K}$ are assumed to be the same for all SSs. $\mathcal{C}_{k}=\left[\mathcal{C}_{k}(0), \ldots, \mathcal{C}_{k}\left(N_{p}-1\right)\right]^{T}$ is the non-zero pilot vector of the $k$-th SS where $\left|\mathcal{C}_{k}(i)\right|=1$ for all $i$ and $k ; D=\frac{N}{N_{p}}$ is an integer representing the pilot spacing within each $\mathrm{SS}$, and $N_{p} \geq L^{\prime}{ }^{1}$. The above preamble design satisfies the best channel identification condition [11] and gives reliable channel gain estimates of all subcarriers. This channel information is useful not only for the data detection in the current frame but also for the optimum resource allocation in the next uplink frame's data transmission. Our considered system does not have any restriction on the data CAS which could be updated through UL-map information message by BS before each uplink frame transmission.

The timing offsets can be absorbed into the CIR as

$$
\boldsymbol{h}_{k}=\left[\mathbf{0}_{d_{k}}^{T}, \overline{\boldsymbol{h}}_{k}^{T}, \mathbf{0}_{N g-d_{k}}^{T}\right]^{T} .
$$

After the CP removal, the time-domain low-pass-equivalent channel output preamble vector $\boldsymbol{s}_{k}$ for the $k$-th SS is given by

$$
\boldsymbol{s}_{k}=\boldsymbol{F}^{H} \overline{\boldsymbol{X}}_{k}\left(\sqrt{N} \boldsymbol{F}_{L} \boldsymbol{h}_{k}\right) \triangleq \boldsymbol{F}^{H} \overline{\boldsymbol{X}}_{k} \boldsymbol{H}_{k} \triangleq \boldsymbol{A}_{k} \boldsymbol{h}_{k}
$$

where $\boldsymbol{H}_{k}=\left[H_{k}(0), H_{k}(1), \ldots, H_{k}(N-1)\right]^{T}$ is the channel frequency response vector for the $k$-th SS, $\boldsymbol{F}$ is the $N \times N$ unitary discrete Fourier transform matrix, $\boldsymbol{F}_{L}$ is the $N \times L$ matrix containing the first $L$ columns of $\boldsymbol{F}$, and $\overline{\boldsymbol{X}}_{k}=$ $\operatorname{diag}\left\{\boldsymbol{X}_{k}\right\}$. The $n$-th sample of $\boldsymbol{s}_{k}$ can be expressed as

$$
s_{k}(n)=\frac{1}{\sqrt{N}} \sum_{i=0}^{N_{P}-1}\left[X_{k}\left(i D+\Delta_{k}\right) H_{k}\left(i D+\Delta_{k}\right)\right] e^{\frac{j 2 \pi\left(i D+\Delta_{k}\right) n}{N}}
$$

${ }^{1}$ Note that the exact knowledge of $L^{\prime}$ may not be readily available. But $N_{g} \geq L^{\prime}$ and $N$ is usually an integer multiple of $N_{g}$ (see IEEE 802.16, 802.16e, [2]). Hence, we simply use $N_{p}=N_{g}$ in the rest of the paper
The corresponding time domain received preamble vector is

$$
\boldsymbol{r}=\sum_{k=1}^{K} \boldsymbol{\Gamma}\left(v_{k}\right) \boldsymbol{s}_{k}+\boldsymbol{z}
$$

where $\boldsymbol{r}=[r(0), r(1), \ldots, r(N-1)]^{T}, \quad \boldsymbol{\Gamma}\left(v_{k}\right)=$ $\operatorname{diag}\left\{\left[1, e^{\frac{j 2 \pi v_{k}}{N}}, \ldots, e^{\frac{j 2 \pi(N-1) v_{k}}{N}}\right]\right\}$, and $\boldsymbol{z}$ is the circularlysymmetric complex Gaussian noise vector with zero mean and covariance matrix $\sigma_{z}^{2} \boldsymbol{I}_{N}$. The signal to noise ratio (SNR) for the $k$-th $\mathrm{SS}$ is defined as

$$
\mathrm{SNR}_{k}=\frac{E\left[\boldsymbol{s}_{k}^{H} \boldsymbol{s}_{k}\right]}{N \sigma_{z}^{2}}
$$

\section{Proposed Modified SAGE Method}

First, we briefly present how the MUI affects the CSAGE (conventional SAGE) method from [10] in term of the signal to interference plus noise ratio (SINR). Then we develop the MSAGE method by incorporating multiuser interference (1) cancellation in both time and frequency domains into the SAGE method and hence by improving the SINR.

Without loss of generality, let us consider the $k$-th SS. In the CSAGE method, the 'complete-data' $\boldsymbol{r}_{k}$ (we will call it 'conventional complete-data') is given by

$$
\boldsymbol{r}_{k}=\boldsymbol{\Gamma}\left(v_{k}\right) \boldsymbol{s}_{k}+\boldsymbol{z}=\boldsymbol{r}-\sum_{j=1, j \neq k}^{K} \boldsymbol{\Gamma}\left(v_{j}\right) \boldsymbol{A}_{j} \boldsymbol{h}_{j} .
$$

Hence, at the $i$-th iteration, the expectation of $\boldsymbol{r}_{k}$ based on the previous estimates $\left\{\hat{v}_{k}^{(i-1)}, \hat{\boldsymbol{h}}_{k}^{(i-1)}\right\}_{k=1}^{K}$ is obtained by

$$
\tilde{\boldsymbol{r}}_{k}^{(i)}=\boldsymbol{\Gamma}\left(v_{k}\right) \boldsymbol{s}_{k}+\sum_{j=1, j \neq k}^{K} \boldsymbol{e}_{j}^{(i)}+\boldsymbol{z}
$$

where $(.)^{(i)}$ denotes the $i$-th iteration, and the interference $\boldsymbol{e}_{j}^{(i)}$ introduced by the $j$-th SS at the $i$-th iteration is due to the estimation error and is given by

$$
\boldsymbol{e}_{j}^{(i)}=\boldsymbol{\Gamma}\left(v_{j}\right) \boldsymbol{A}_{j} \boldsymbol{h}_{j}-\boldsymbol{\Gamma}\left(\hat{v}_{j}^{(i-1)}\right) \boldsymbol{A}_{j} \hat{\boldsymbol{h}}_{j}^{(i-1)} .
$$

Then the joint ML estimation of the parameters for the $k$-th SS is performed based on $\tilde{\boldsymbol{r}}_{k}^{(i)}$. Obviously, in addition to the desired signal and the Gaussian noise, MUI is also involved in $\tilde{\boldsymbol{r}}_{k}$. The performance of the ML estimator at the $i$-th iteration depends on the SINR at the $i$-th iteration which is given by

$$
\operatorname{SINR}_{k}^{(i)}=\frac{E\left[\boldsymbol{s}_{k}^{H} \boldsymbol{s}_{k}\right]}{\sum_{j=1, j \neq k}^{K} E\left[\left(\boldsymbol{e}_{j}^{(i)}\right)^{H} \boldsymbol{e}_{j}^{(i)}\right]+N \sigma_{z}^{2}} .
$$

$E\left[\boldsymbol{e}_{j}^{(i), H} \boldsymbol{e}_{j}^{(i)}\right]$ in (10) is the average interference power from the $j$-th SS and is given by

$$
E\left[\left(\boldsymbol{e}_{j}^{(i)}\right)^{H} \boldsymbol{e}_{j}^{(i)}\right] \approx a_{j}^{2} \sigma_{h}^{2} N_{p}\left[2+\rho_{h}^{(i)}-2 \Re\left(\alpha\left(\Delta v_{j}^{(i)}\right)\right)\right]
$$

where $\sigma_{h}^{2}=E\left[\boldsymbol{h}_{k}^{H} \boldsymbol{h}_{k}\right] ; \Delta v_{j}^{(i)}=v_{j}-\hat{v}_{j}^{(i-1)}$ and $\rho_{h}^{(i)}=$ $\frac{\sum_{n=0}^{L-1} E\left[\left|h(n)-\hat{h}^{(i-1)}(n)\right|^{2}\right]}{\sigma_{h}^{2}}$ are the frequency offset estimation error (the residual frequency offset) and the normalized channel estimation mean square error, respectively, at the beginning of the $i$-th iteration. $\alpha(v+n-m)=\frac{1}{N} \sum_{p=0}^{N-1} e^{\frac{j 2 \pi(v+n-m)_{p}}{N}}$ is the ICI coefficient corresponding to the interference from 
the $n$-th tone to the $m$-th tone when the frequency offset is equal to $v$.

The effects of the number of SSs $K$, the estimation errors of the previous iteration, and SNR on the $\operatorname{SINR}_{k}^{(i)}$ of the CSAGE method are presented in Fig. 1(a). For illustration purpose, we assume that all SSs have the same $\Delta v_{j}^{(i)}$ and $\rho_{h}^{(i)}$ (denoted by $\Delta v$ and $\rho_{h}$ in the figure). $\operatorname{SINR}_{k}^{(i)}$ of the CSAGE method degrades significantly when $K$ and/or the previous iteration estimation errors increase. In particular, when $\Delta v=0.1, \rho_{h}=$ 0.01 and $K=8, \operatorname{SINR}_{k}^{(i)}$ is about $20 \mathrm{~dB}$ lower than SNR. Increasing SNR does not improve $\operatorname{SINR}_{k}^{(i)}$ much when the previous iteration estimation errors are relatively large (say, $\Delta v>0.1$ and $\rho_{h}=0.01$ ), which usually occur at the first several iterations of the CSAGE algorithm resulting in a slower convergence rate as well as unreliable estimation performance.

Different from the CSAGE method which improves SINR iteratively through MUI cancelation in time-domain, we propose a modified method which improves SINR by suppressing MUI in both time and frequency domain as follows. We perform two steps prior to the parameters estimation of the $k$-th SS. First, we perform frequency offset compensation of the $k$-th SS in the time-domain using previous frequency offset estimate so that the transmitted energy of each pilot is more concentrated at the corresponding subcarrier. Next, based on the proposed interleaved pilot structure, we perform frequency-domain windowing centered at each of the $N_{p}$ pilots of the $k$-th SS in order to suppress interference from other SSs while keeping most of $k$-th SS's signal energy within the window. Each window has a bandwidth of $B$ subcarriers $^{2}$ and is symmetric at the corresponding subcarrier.

After these two steps, we define a 'new complete data' in frequency-domain as

$$
\boldsymbol{Y}_{k}=\boldsymbol{\Phi}_{k} \boldsymbol{F} \boldsymbol{\Gamma}^{H}\left(v_{k}\right) \boldsymbol{r}_{k}=\boldsymbol{\Phi}_{k} \boldsymbol{F} \boldsymbol{s}_{k}+\boldsymbol{\Phi}_{k} \boldsymbol{F} \boldsymbol{z}
$$

where $\boldsymbol{\Phi}_{k}$ represents the frequency-domain windowing operation for the $k$-th SS and its $m$-th row, $n$-th column element is given by

$$
\boldsymbol{\Phi}_{k}(m, n)= \begin{cases}1, & (m=n)\left(m=\bmod \left(i D+\Delta_{k} \pm b, N\right)\right. \\ & \left.i=0, \ldots, N_{p}-1 ; b=0, \ldots, \frac{B-1}{2}\right) \\ 0, & \text { otherwise }\end{cases}
$$

Note that the corresponding time-domain signal is not the same as the 'conventional complete-data' $\boldsymbol{r}_{k}$.

The expectation step of the modified SAGE method at the $i$-th iteration is given by

$$
\begin{aligned}
& \tilde{\boldsymbol{Y}}_{k}^{(i)}=\boldsymbol{F} \boldsymbol{\Gamma}\left(\Delta v_{k}^{(i)}\right) \boldsymbol{s}_{k}+\underbrace{\left[\boldsymbol{\Phi}_{k} \boldsymbol{F} \boldsymbol{\Gamma}\left(\Delta v_{k}^{(i)}\right)-\boldsymbol{F} \boldsymbol{\Gamma}\left(\Delta v_{k}^{(i)}\right)\right] \boldsymbol{s}_{k}}_{\tilde{s}_{k}^{(i)}} \\
& +\sum_{j=1, j \neq k}^{K} \underbrace{\boldsymbol{\Phi}_{k} \boldsymbol{F} \boldsymbol{\Gamma}^{H}\left(\hat{v}_{k}^{(i-1)}\right) \boldsymbol{e}_{j}^{(i)}}_{\tilde{I}_{j}^{(i)}}+\underbrace{\boldsymbol{\Phi}_{k} \boldsymbol{F} \boldsymbol{\Gamma}^{H}\left(\hat{v}_{k}^{(i-1)}\right) \boldsymbol{z}}_{W_{k}^{(i)}}
\end{aligned}
$$

where $\tilde{\boldsymbol{S}}_{k}^{(i)}$ is the signal leakage outside the $k$-th SS's frequency-domain windows. $\tilde{\boldsymbol{I}}_{j}^{(i)}$ and $\boldsymbol{W}_{k}^{(i)}$ are the interference introduced by the $j$-th SS and the Gaussian noise within in

\footnotetext{
${ }^{2} B$ is a positive odd integer.
}

the $k$-th SS's windows, respectively. From (14), we observe that $\boldsymbol{Y}_{k}$ is no longer the 'complete-data' for $v_{k}$ but for the frequency offset estimation error $\Delta v_{k}$.

The SINR of the new complete data $\tilde{\boldsymbol{Y}}_{k}^{(i)}$ at the $i$-th iteration is given by

$$
\begin{aligned}
& \widetilde{\operatorname{SINR}}_{k}^{(i)}=\frac{E\left[\boldsymbol{s}_{k}^{H} \boldsymbol{s}_{k}\right]}{\sum_{j=1, j \neq k}^{K} E\left[\left(\tilde{\boldsymbol{I}}_{j}^{(i)}\right)^{H} \tilde{\boldsymbol{I}}_{j}^{(i)}\right]+N \sigma_{z}^{2} B / D+E\left[\tilde{\boldsymbol{S}}_{k}^{H,(i)} \tilde{\boldsymbol{S}}_{k}^{(i)}\right]} \\
& \text { where } E\left[\left(\tilde{\boldsymbol{I}}_{j}^{(i)}\right)^{H} \tilde{\boldsymbol{I}}_{j}^{(i)}\right] \approx \frac{a_{j}^{2} \sigma_{h}^{2}}{D^{2}} \sum_{n=0}^{N-1} \sum_{p \in J_{n}} \beta(p) \gamma^{2}(p) \\
& \left\{e ^ { \frac { j 2 \pi ( \Delta _ { j } - \Delta _ { k } ) p } { D } } \left[1+\left(1+\rho_{h}^{(i)}\right) e^{\frac{-j 2 \pi \Delta v_{j}^{(i)} p}{D}}\right.\right. \\
& \left.\left.-e^{\frac{-j 2 \pi\left(n+p N_{p}\right) \Delta v_{j}^{(i)}}{N}}-e^{\frac{j 2 \pi n \Delta v_{j}^{(i)}}{N}}\right]\right\} \\
& E\left[\left(\tilde{\boldsymbol{S}}_{k}^{(i)}\right)^{H} \tilde{\boldsymbol{S}}_{k}^{(i)}\right]=a_{k}^{2} \sigma_{h}^{2}\left[N_{p}-\frac{1}{D^{2}} \sum_{n=0}^{N-1} \sum_{p \in J_{n}} \beta(p) e^{\frac{j 2 \pi \Delta v_{k}^{(i)} p}{D}}\right] \\
& \beta(p) \triangleq \sum_{q=-\frac{B-1}{2}}^{\frac{B-1}{2}} e^{\frac{-j 2 \pi q p}{D}}= \begin{cases}B, & p=0 \\
\frac{\sin \left(\frac{\pi B p}{D}\right)}{\sin \left(\frac{\pi p}{D}\right)}, & \text { otherwise }\end{cases} \\
& \gamma(p) \triangleq E\left[e^{\frac{-j 2 \pi p v}{D}}\right]= \begin{cases}1, & p=0 \\
\frac{D}{p \pi} \sin \left(\frac{\pi p}{D}\right), & \text { otherwise }\end{cases} \\
& J_{n} \triangleq\left\{\left\lceil-n / N_{P}\right\rceil,\left\lceil-n / N_{P}\right\rceil+1, \ldots,\left\lfloor(N-1-n) / N_{P}\right\rfloor\right\} .
\end{aligned}
$$

Different from (10), $\widetilde{\operatorname{SINR}}_{k}^{(i)}$ depends on not only the estimation errors and SNR but also the window bandwidth $B$. The optimum choice of $B$ at the $i$-th iteration is obtained by maximizing (15) as

$$
B^{\dagger,(i)}=\underset{B}{\arg \max }\left\{\widetilde{\operatorname{SINR}}_{k}^{(i)}\right\}
$$

Unfortunately, no closed-form solution for the exact optimum $B$ could be obtained due to the intractable optimization problem and the lack of the knowledge of the exact estimation errors. However, for given system parameters $\left(N, N_{p}, K\right)$ and fixed design values of the estimation errors, $B^{\dagger,(i)}$ could be numerically obtained.

Fig. 2 shows the effect of $B$ on the SINR for several values of $K, \mathrm{SNR}$ and the estimation errors. Smaller $K$ or/and larger estimation errors of the previous iteration give a larger optimum $B$. For a small $K$, the optimum $B$ increases with SNR. If a fixed $B$ is used throughout the proposed algorithm, $B=3$ would be a good choice. Alternatively, we can use an adaptive $B$. We can set the initial value of $B$ by utilizing the knowledge of $K$ and the operating SNR that the BS has, together with (relatively large) designed values of initial estimation errors. Then after a few iterations as the estimation errors decrease, we can choose a smaller $B$.

Fig. 1(b) shows the $\widetilde{\operatorname{SINR}}_{k}^{(i)}$ versus $K$ for several values of SNR and estimation errors. Although using a fixed $B$ gives a slight SINR difference between small and large values of $K$ at high SNR (20 dB), the SINR differences are negligible at typical SNR values (say $10 \mathrm{~dB}$ ). This fact justifies the use of a fixed $B$. By comparing Fig. 1(a) and (b), we observe that the SINR improvement of our proposed MSAGE method over the CSAGE method is about $10 \mathrm{~dB}$ in all conditions. Our proposed MSAGE algorithm using the 'new complete-data' is summarized as follows: 
- E-step: For $i=1,2, \ldots$, and $k=\bmod (i, K)$, compute

$$
\tilde{\boldsymbol{Y}}_{k}^{(i)}=\mathfrak{B}_{\boldsymbol{\Phi}_{k}}\left\{\boldsymbol{F} \boldsymbol{\Gamma}^{H}\left(\hat{v}_{k}^{(i-1)}\right)\left[\boldsymbol{r}-\sum_{j=1, j \neq k}^{K} \boldsymbol{\Gamma}\left(\hat{v}_{j}^{(i-1)}\right) \boldsymbol{A}_{j} \hat{\boldsymbol{h}}_{j}^{(i-1)}\right]\right\} .
$$

- M-step: Compute

$$
\begin{aligned}
&\left\{\Delta \hat{v}_{k}^{(i)}, \hat{\boldsymbol{h}}_{k}^{(i)}\right\}= \underset{\Delta \tilde{v}_{k}, \tilde{\boldsymbol{h}}_{k}}{\arg \min }\left\{\left\|\tilde{\boldsymbol{Y}}_{k}^{(i)}-\mathfrak{B}_{\Phi_{k}}\left\{\boldsymbol{F} \boldsymbol{\Gamma}\left(\Delta \tilde{v}_{k}\right) \boldsymbol{A}_{k} \tilde{\boldsymbol{h}}_{k}\right\}\right\|\right\}(23) \\
& \hat{v}_{k}^{(i)}= \hat{v}_{k}^{(i-1)}+\Delta \hat{v}_{k}^{(i)} . \\
& \text { For } j \neq k, \quad\left\{\hat{v}_{j}^{(i)}, \hat{\boldsymbol{h}}_{j}^{(i)}\right\}=\left\{\hat{v}_{j}^{(i-1)}, \hat{\boldsymbol{h}}_{j}^{(i-1)}\right\} .
\end{aligned}
$$

Solving the above optimization in (23) gives

$$
\begin{aligned}
& \Delta \hat{v}_{k}^{(i)}=\underset{\Delta \tilde{v}_{k}}{\arg \max } \Re\left\{\left(\tilde{\boldsymbol{Y}}_{k}^{(i)}\right)^{H} \boldsymbol{\Omega}\left(\Delta \tilde{v}_{k}\right) \Upsilon\left(\Delta \tilde{v}_{k}\right) \tilde{\boldsymbol{Y}}_{k}^{(i)}\right\} \\
& \text { and } \hat{\boldsymbol{h}}_{k}^{(i)}=\boldsymbol{\Upsilon}\left(\Delta \hat{v}_{k}^{(i)}\right) \tilde{\boldsymbol{Y}}_{k}^{(i)} \\
& \text { where } \boldsymbol{\Omega}\left(\Delta \tilde{v}_{k}\right)=\mathfrak{B}_{\boldsymbol{\Phi}_{k}}\left\{\boldsymbol{F} \boldsymbol{\Gamma}\left(\Delta \tilde{v}_{k}\right) \boldsymbol{A}_{k}\right\} \\
& \mathbf{\Upsilon}\left(\Delta \hat{v}_{k}^{(i)}\right)=\left(\boldsymbol{\Omega}^{H}\left(\Delta \hat{v}_{k}^{(i)}\right) \boldsymbol{\Omega}\left(\Delta \hat{v}_{k}^{(i)}\right)\right)^{-1} \boldsymbol{\Omega}^{H}\left(\Delta \hat{v}_{k}^{(i)}\right)
\end{aligned}
$$

The above estimators have smaller complexity than the counterparts in the CSAGE method due to the smaller sizes of matrices and vectors involved. The initial estimates $\left\{\hat{v}_{k}^{(0)}\right\}$ are set to zeros (the mean value of $\left\{\hat{v}_{k}^{(0)}\right\}$ ), and $\left\{\hat{\boldsymbol{h}}_{k}^{(0)}\right\}$ are obtained from (27) with $\Delta \hat{v}_{k}^{(0)} \triangleq 0$ and $\tilde{\boldsymbol{Y}}_{k}^{(0)} \triangleq \mathfrak{B}_{\boldsymbol{\Phi}_{k}}\{\boldsymbol{F r}\}$.

\section{Proposed Ad Hoc Iterative Method}

Our proposed MSAGE method converges much faster than the CSAGE method due to the SINR improvement and also has lower complexity. But the MSAGE's frequency offset estimator in (26) still requires a large complexity. In this section, we present a much lower complexity estimator set to replace the M-step ((26) and (27)) of the MSAGE method.

First, the expectation of the 'new complete-data' in the timedomain at the $i$-th iteration can be expressed as

$$
\tilde{\boldsymbol{y}}_{k}^{(i)}=\boldsymbol{\Gamma}\left(\Delta v_{k}^{(i)}\right) \boldsymbol{s}_{k}+\underbrace{\boldsymbol{F}^{H}\left[\sum_{j=1, j \neq k}^{K} \tilde{\boldsymbol{I}}_{j}^{(i)}+\tilde{\boldsymbol{S}}_{k}^{(i)}+\boldsymbol{W}_{k}^{(i)}\right]}_{\overline{\boldsymbol{w}}_{k}^{(i)}} .
$$

Therefore, the $n$-th sample of $\tilde{\boldsymbol{y}}_{k}^{(i)}$ could be expressed as

$$
\tilde{y}_{k}^{(i)}(n)=e^{\frac{j 2 \pi n \Delta v_{k}^{(i)}}{N}}\left[s_{k}(n)+\bar{w}_{k}^{(i)}(n)\right] .
$$

Note that due to our proposed pilot design, $\boldsymbol{s}_{k}$ contains $D$ identical (if certain known phase shifts are compensated) parts of length $N_{p}$ each. We utilize this periodicity property of the training signals together with SINR $\gg 1$ in developing our ad hoc method. Define the correlation terms as

$$
\begin{aligned}
& R_{k}^{(i)}(m)=\frac{1}{N-m N_{p}} \sum_{n=0}^{N-m N_{p}-1}\left(\tilde{y}_{k}^{(i)}(n)\right)^{*} \tilde{y}_{k}^{(i)}\left(n+m N_{p}\right) \\
= & e^{\frac{j 2 \pi m\left(\Delta_{k}+\Delta v_{k}^{(i)}\right)}{D}} E_{s, k}\left[1+\gamma_{k}^{(i)}(m)\right], m=0,1, \ldots, M-1
\end{aligned}
$$

where

$$
\begin{aligned}
& E_{s, k}=\frac{1}{N_{p}} \sum_{n=0}^{N_{p}-1}\left|s_{k}(n)\right|^{2} \\
& \gamma_{k}^{(i)}(m)=\frac{e^{\frac{-j 2 \pi m \Delta_{k}}{D}}}{\left(N-m N_{P}\right) \varepsilon_{s}^{2}} \sum_{n=0}^{N-m N_{p}-1}\left[s_{k}^{*}(n) \bar{w}_{k}^{(i)}\left(n+m N_{p}\right)\right. \\
& \left.+s_{k}\left(n+m N_{p}\right)\left(\bar{w}_{k}^{(i)}(n)\right)^{*}+\left(\bar{w}_{k}^{(i)}(n)\right)^{*} \bar{w}_{k}^{(i)}\left(n+m N_{p}\right)\right](33)
\end{aligned}
$$

and $M$ is a design parameter satisfying $M \leq \frac{D}{2}$. Next, define

$$
\begin{aligned}
\varphi_{k}^{(i)}(m) & =\arg \left\{R_{k}^{(i)}(m)\left(R_{k}^{(i)}(m-1)\right)^{*}\right\} \\
& \approx \frac{2 \pi\left(\Delta v_{k}^{(i)}+\Delta_{k}\right)}{D}+\gamma_{I, k}^{(i)}(m)-\gamma_{I, k}^{(i)}(m-1)(34)
\end{aligned}
$$

where $\gamma_{I, k}^{(i)}(m)$ is the imaginary part of $\gamma_{k}^{(i)}(m)$. The above approximation holds when $\widehat{\operatorname{SINR}}_{k}^{(i)} \gg 1$ (our method satisfies this requirement, c.f. Fig. 1). Developing the best linear unbiased estimator [12] is infeasible in this case because the exact values of the frequency offsets are required in the covariance matrix calculation. Hence, we apply the least-squares (LS) principle to (34) and obtain our ad hoc frequency offset estimator as

$$
\Delta \hat{v}_{k}^{(i)}=\frac{D}{2 M \pi} \sum_{m=1}^{M} \varphi_{k}^{(i)}(m)-\Delta_{k}
$$

Performing the frequency offset compensation using the above estimate and applying the LS channel estimation, we obtain the channel estimate for the $k$-th SS at the $i$-th iteration as

$$
\hat{\boldsymbol{h}}_{k}^{(i)}=\left(\boldsymbol{A}_{k}^{H} \boldsymbol{A}_{k}\right)^{-1} \boldsymbol{A}_{k}^{H} \boldsymbol{\Gamma}^{H}\left(\hat{v}_{k}^{(i)}\right) \tilde{\boldsymbol{y}}_{k}^{(i)} \text {. }
$$

In summary, our proposed ad hoc iterative method is obtained by replacing (22), (26) and (27) with (30), (35) and (36), respectively.

\section{Simulation Results and Discussions}

The simulation parameters are selected from [2]. Uplink bandwidth is $2.5 \mathrm{MHz}$ corresponding to sampling period $T_{s}=0.35 \mu \mathrm{s}$. The sub-carrier spacing is $11.16 \mathrm{kHz}$. The system has $N=256$ sub-carriers unless mentioned otherwise. The maximum timing offset $d_{\text {max }}$ is set to 8 samples $^{3} . N_{g}$ is set to 16. Actual timing offsets (in samples) are generated with a discrete uniform distribution within the range $[0,8] .{ }^{4}$ The frequency offsets $\left\{v_{k}\right\}$ are independently generated with a uniform distribution within $[-0.5,0.5]$. The SUI-3 channel model with 3 paths [13] is used. The CIR including the combined transmit and receive filter effect (before incorporating the timing offset) for the $k$-th user is given by

$$
\bar{h}_{k}(l)=\sum_{i=0}^{2} \zeta_{i} g_{T}\left(l T_{s}-\tau_{i}-t_{0}\right), l=0, \ldots, L-1
$$

where $g_{T}(t)$ is a spectral raised-cosine filter with a roll-off factor of $0.5,\left\{\zeta_{i}\right\}$ are the complex Gaussian channel path gains, $\left\{\tau_{i}\right\}$ are the the channel path delays, and $t_{0}$ is a time shift for causality. The average total energy of the channel taps $\sigma_{h}^{2}$ for each user is set to unity and $L$ is set to 7 .

The proposed preamble used in the AH and MSAGE methods follows (1) with $N_{p}=16$. During data transmission of the proposed methods, arbitrary (random) CAS is used where $N$ sub-carriers are divided into $Q=K$ sub-channels and each sub-channel has $P=N / K$ sub-carriers (corresponding to a fully-loaded system). For the reference CSAGE method, an

\footnotetext{
${ }^{3}$ It satisfies the synchronization requirement $( \pm 1 / 4$ of the minimum $\mathrm{CP})$ defined in the IEEE 802.16-2004

${ }^{4}$ Non-integer part of the timing offset can be absorbed into the CIR and hence, we just use integer timing offsets (in samples) in the simulation.
} 
arbitrary CAS is used (each SS uses $P$ sub-carriers too) but is kept the same for both the preamble and the data transmission. For the fixed $B$ approach, we use $B=3$ sub-carriers. For the ad hoc frequency estimator in (35), $M$ is set to $\frac{N}{2 N_{p}}$ for all SSs. For the CSAGE and MSAGE methods, the number of grid points $N_{s}$ for frequency offset estimator is set to 201. Unless mentioned otherwise, all the simulation results are based on the 10000 simulation runs.

Figs. 3-5 show the mean-square error (MSE) and uncoded bit error rate (BER) performance of the AH, MSAGE and CSAGE methods versus the number of iterations for different values of $K$. Note that we count $K$ iterations ( $i$-loop in the summarized description of the MSAGE algorithm) as one iteration in the figures since only one SS is updated each time and there are $K$ SSs.

1) MSE Performance of Frequency Offset Estimators: Fig. 3 and Fig. 4 show the MSE performances of the frequency offset estimators from the CSAGE, MSAGE, and AH methods for $K=4$ and $K=8$, respectively. Both the convergence rate and the MSE of the CSAGE method degrade significantly when $K$ increases from 4 to 8 . The reason is that the increase in $K$ enlarges the MUI and results in a lower SINR. Our proposed AH and MSAGE methods experience no noticeable performance degradation when $K$ increases (the increase in $K$ only makes the proposed two methods converge slower at the first two iterations). Both proposed methods need less number of iterations than the CSAGE method to achieve the same performance. The proposed methods also achieve similar improvement in channel estimation but corresponding simulation results are excluded due to space limitation.

2) Uncoded BER Performance: QPSK data modulation is used and all BER results are obtained from 1000 simulation runs. Fig. 5 shows the BER curves for $K=8$. The BERs of the proposed AH and MSAGE methods are much closer to the ideal performance obtained with known frequency offsets and channel responses than those of the CSAGE method due to the better synchronization and channel estimation performance.

3) Adaptive B Effect: Fig. 6 illustrates the performance of the proposed frequency offset estimator using an adaptive $B$ in an OFDMA system with $N=1024$ subcarriers and $K=2$ users. The parameter $B_{a d p t}$ is set to 33 at the first iteration and changed to 9 at the beginning of the second iteration. If compared with fixed- $B$ approaches, the adaptive $B$ approach achieves a slight advantage since the large $B$ at the first iteration yields a faster convergence while the small $B$ at later iterations gives better performance.

4) Computation Complexity: The overall computation load of iterative methods consist of two parts: computation complexity at each iteration and the number of iterations used. At each iteration, the CSAGE method needs $\mathcal{O}\left(K\left(N_{s}\left(2 N N_{g}+\right.\right.\right.$ $\left.N)+N N_{g}\right)$ ) complex operations due to $N \times N_{g}$ dimension matrix multiplication at each grid point. For the MSAGE method, only $B N_{p} \times N_{g}$ matrix multiplication is needed at each grid point (the matrix $\boldsymbol{\Omega}_{k}(v)$ and $\boldsymbol{\Upsilon}_{k}(v)$ can be precomputed and stored) so that its computation complexity is reduced to $\mathcal{O}\left(K\left(N_{s}\left(2 B N_{p} N_{g}+B N_{p}\right)+N \log _{2} N+\right.\right.$
$\left.\left.B N_{g} N_{p}\right)\right)$ at each iteration. The $\mathrm{AH}$ method needs the smallest computation complexity per iteration which is $\mathcal{O}(K(M N+$ $\left.2 N \log _{2} N+N N_{g}\right)$ ). For our simulation parameters, the order of complex operations for the CSAGE, MSAGE and AH methods are approximately $1702144 K, 321200 K$, and $10240 K$, respectively, where $K$ is the number of users. Moreover, the CSAGE method needs more iterations to converge. Hence, both proposed methods have much less computation load than the CSAGE method.

\section{CONClusions}

We have presented two iterative methods for the joint estimation of frequency offsets and channels in OFDMA uplink by using disjoint sets of cyclically equal-spaced, equal energy pilot tones assigned to the uplink users. The first method (MSAGE) incorporates multiuser interference cancellation in both time and frequency domains into the SAGE method. The second method $(\mathrm{AH})$ replaces the complexity-intensive M-step of the MSAGE method with a significantly lower complexity version. By utilizing the time-domain characteristics of the proposed pilot signals, the frequency-domain power concentration property of OFDMA systems, and the multiuser interference cancellation in both time and frequency domains, both proposed methods achieve faster convergence rate, better estimation performance, lower complexity, and better robustness against the number of users than the existing conventional SAGE method. The AH method is very appealing for practical implementation since it achieves almost the same performance as the MSAGE method while requiring much lower complexity.

\section{REFERENCES}

[1] IEEE LAN/MAN Standards Committee, "Broadband Wireless Access: IEEE MAN standard," IEEE 802.16-2004, 2004.

[2] H.Yaghoobi,"Scalable OFDMA physical layer in IEEE 802.16 WirelessMAN," Intel Technology Journal., Vol. 8, No. 3, Aug. 2004, pp. 201-212.

[3] H. Minn and X. Fu,"A new ranging method for OFDMA systems," IEEE GLOBECOM 2005,, Vol. 3, pp. 1435-1440.

[4] T. Pollet, M. Van Bladel and M. Moeneclaey, "BER sensitivity of OFDM systems to carrier frequency offset and Wiener phase noise," IEEE Trans. Commun., Vol. 43, No. 2/3/4, Feb/Mar/Apr. 1995, pp. 191-193.

[5] M. Gudmundson and P. O. Anderson, "Adjacent channel interference in an OFDM system," Proc. IEEE VTC 1996, pp. 918-922.

[6] M. Morelli, "Timing and frequency synchronization for the uplink of an OFDMA System," IEEE Trans. Commun., No. 2, Feb. 2004, pp. 296-306.

[7] J.J.van de Beek, P. O. Borjesson, M. L. Bouchest, D. Landstram, J.M. Arenas, P. Odling, C. Ostberg, M. Wahlqvist, and S.K.Wilson, "A time and frequency synchronization scheme for multiuser OFDM," IEEE $J$. Select. Areas Commun., Vol. 17, pp. 1900-1914, Nov.1999.

[8] Z. Cao, U. Tureli, Y.D. Yao, "Efficient structure-based carrier frequency offset estimation for interleaved OFDMA uplink," Proc. IEEE ICC 2003, pp.3361-3365.

[9] Man-On Pun, Shang-Ho Tsai and C.-C.Jay Kuo, "Joint maximum likelihood estimation of carrier frequency offset and channel in uplink OFDMA systems," IEEE Globecom 2004, pp. 3748-3752.

[10] —, "An EM-based joint maximum likelihood estimation of carrier frequency offset and channel for uplink OFDMA systems," IEEE VTC 2004, pp. 598-602.

[11] H. Minn and N. Al-Dhahir, "Optimal training signals for MIMO OFDM channel estimation," IEEE Globecom 2004, pp. 219-224.

[12] M.Morelli and U.Mengali, "An improved frequency offset estimator for OFDM applications ," IEEE Commun Letters, vol.3,pp.75-77, Mar.1999

[13] IEEE LAN/MAN Standards Committee, "Channel models for fixed wireless applications," Document IEEE.802.16.3c-01/29r4. 
(a)

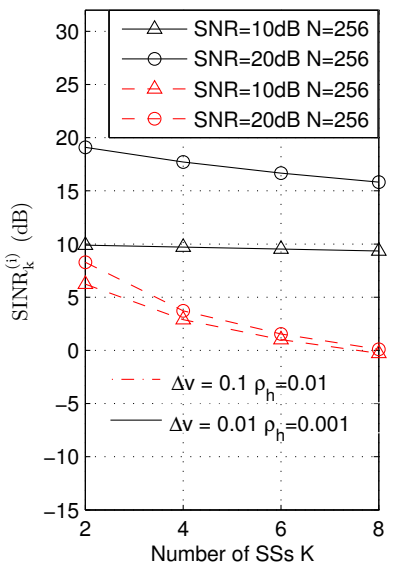

(b)



Fig. 1. The impact of $K, \mathrm{SNR}, \Delta v$, and $\rho_{\boldsymbol{h}}$ on SINR for (a) the CSAGE method, (b) the MSAGE method $(B=3)$


Fig. 2. The effect of $B$ on SINR under different SNR, $K, \Delta v$, and $\rho_{h}$

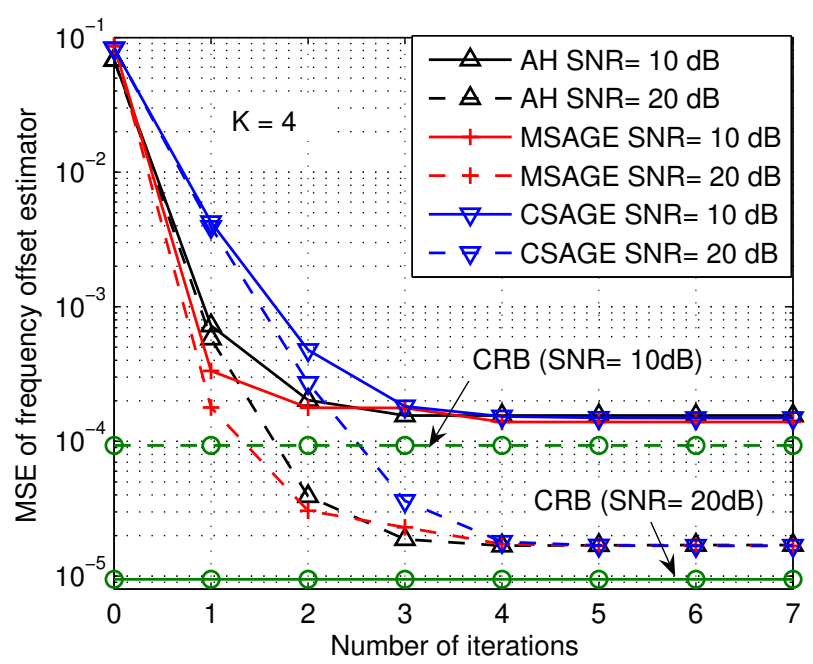

Fig. 3. MSE comparison of frequency offset estimators $(K=4)$

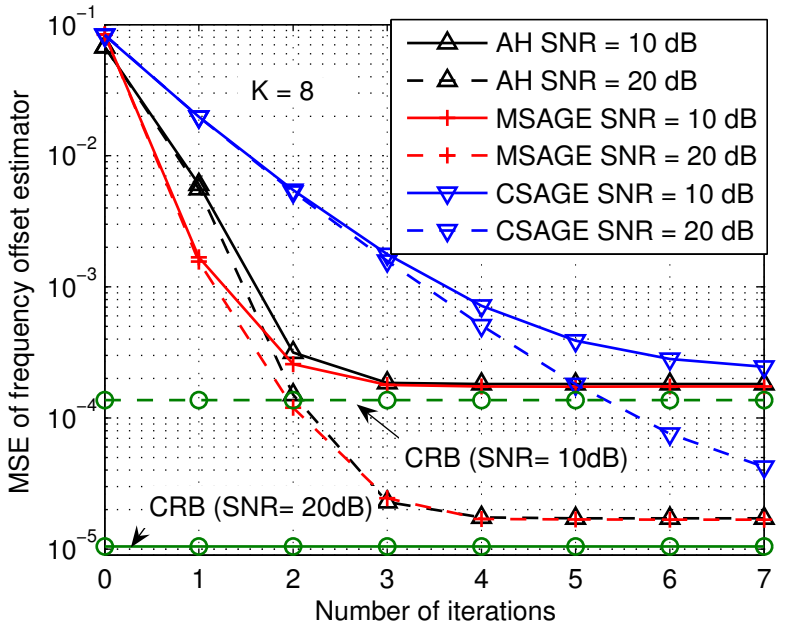

Fig. 4. MSE comparison of frequency offset estimators $(K=8)$

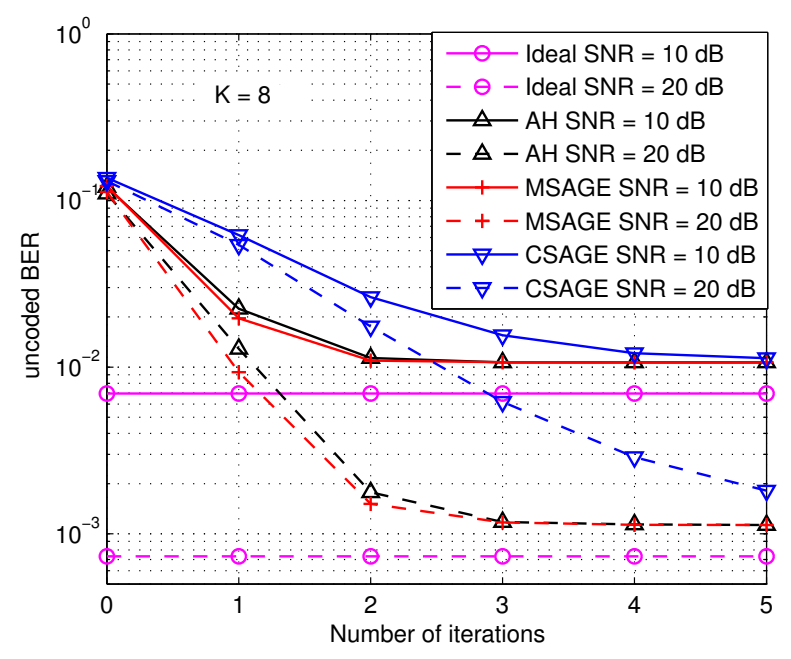

Fig. 5. Uncoded BER comparison



Fig. 6. MSE performance of the proposed frequency offset estimator using fixed- $B$ and adaptive $B$ 